\title{
Reversion of Yeast Protoplasts in Media containing Polyethylene Glycol
}

\author{
By A. SVOBODA* AND D. PIEDRA $\dagger$ \\ Department of Biology, Faculty of Medicine, J. E. Purkyně University, 66243 Brno, \\ Czechoslovakia
}

(Received 21 February 1983; revised 27 June 1983)

\begin{abstract}
Liquid medium supplemented with $35 \%$ (w/v) PEG supported regeneration of the cell wall in protoplasts of Saccharomyces cerevisiae. The regeneration was followed by reversion to normal cells. Both these morphogenetic processes were similar to those described previously in gelatin media. The frequency of reversion was estimated to be 20 to $30 \%$. The regeneration medium with PEG also induced protoplast fusion. When complementary auxotrophs were used for fusion only the fused products proliferated, giving first regenerated protoplasts and then hybrid cells.
\end{abstract}

\section{INTRODUCTION}

Reversion of yeast protoplasts to cells is strictly dependent on the formation of complete new cell walls on their surfaces. Protoplasts do not revert in media which cannot support complete cell wall formation; instead, they grow into tubular vacuolated bodies with limited viability (Nečas, 1971). Structurally and functionally regenerated walls are produced when protoplasts are embedded in gel media containing gelatin (Nečas, 1961) or agar (Svoboda, 1966). Although protoplasts of some species may regenerate walls in liquid media, regeneration in gels is more efficient and faster (for a review see Nečas \& Svoboda, 1981).

The use of concentrated gelatine ( 30 to $40 \%, w / v)$ or quickly solidifying agar ( 2 to $3 \%$, w/v) often results in imperfect embedding and, consequently, in a decrease in reversion frequency. Also, isolation of regenerating protoplasts from gels, particularly from agar, is difficult. Moreover, gelatin, due to its complex composition, cannot be used for cultivating most of the auxotrophs. All these reasons led investigators to seek other regeneration media that would be well defined, easy to use and suitable for genetic and biochemical analyses.

In studies on PEG-induced fusion of Saccharomyces cerevisiae protoplasts, Darling (1976) noticed that occasionally protoplasts reverted to cells in medium containing the fusogen. On the basis of this finding we made a thorough study of the effect of PEG on cell wall regeneration, reversion to cells and protoplast fusion. Our results confirm that PEG-supplemented media support cell wall regeneration in yeast protoplasts as efficiently as do the gel media.

\section{METHODS}

Strains. The following strains of Saccharomyces cerevisiae were used: diploid wild-type CCY 21-4-59, haploid strain 9 his $\alpha$ and 55R5-3C ura $\alpha$, kindly provided by Dr M. Opekarová and Dr J. Subík.

Culture media. All the strains were maintained on wort-agar slants. Cells were grown in malt extract medium, protoplasts were cultured in either minimal glucose medium (minimal medium; Leupold, 1955) or in Difco Nitrogen Base-glucose medium (YNBG medium). Both media were supplemented with $0.6 \mathrm{M}-\mathrm{KCl}$ (as an osmotic stabilizer) and/or PEG. In some experiments, medium containing 3\%(w/v) yeast extract (Difco) and glucose was used (YEG medium). When required PEG 4000 or 6000 was included at concentrations ranging from 10 to $40 \%$ $(\mathrm{w} / \mathrm{v})$.

Protoplast preparation and cultivation. A modified method of Eddy \& Williamson (1959), using lyophilized snail enzymes and $0.6 \mathrm{M}-\mathrm{KCl}$, was employed. Freshly prepared protoplasts (yields over $99 \%$ ) were washed free from the

† Present address: National Centre for Scientific Research, P.O. Box 6990, Havana, Cuba. 
snail enzymes by $0.6 \mathrm{M}-\mathrm{KCl}$. For regeneration experiments, pellets of protoplasts were resuspended $\left(10^{2}\right.$ to $10^{6} \mathrm{ml}^{-1}$ ) in regeneration medium, mixed well on a vortex agitator and incubated at $28^{\circ} \mathrm{C}$.

Reversion of fused protoplasts. Equal volumes of suspensions containing $10^{6}$ protoplasts of $S$. cerevisiae haploid strains 9 and 55R5-3C were mixed and centrifuged. The pellet was resuspended in $10 \mathrm{ml}$ PEG-minimal medium, thoroughly shaken on a vortex agitator and incubated on a plate at $28^{\circ} \mathrm{C}$ for $48 \mathrm{~h}$. The suspension was then washed from PEG and the pellet was seeded on agar minimal medium plates and incubated at $28^{\circ} \mathrm{C}$.

Isolation of regenerating wall material. Samples $(5 \mathrm{ml})$ of protoplast suspension in PEG media were withdrawn at intervals, mixed with $5 \mathrm{ml}$ distilled water and centrifuged. The pellet was washed with distilled water three times. The pelleted material was analysed for carbohydrates and dry weight (Chung \& Nickerson, 1954). A portion of the suspension was taken for electron microscopic examination either directly or after treatment with $0.5 \%(w / v)$ SDS for $30 \mathrm{~min}$ at $50^{\circ} \mathrm{C}$ (Kopecká \& Farkaš, 1979).

Electron microscopy. Samples of wall material were transferred on to formvar-coated grids and shadowed with platinum at an angle of $25^{\circ}$. For freeze-etched replicas, samples were withdrawn from the protoplast culture at intervals, briefly centrifuged and frozen in Freon 22 cooled with liquid nitrogen. Replicas were made in a Balzers freeze-fracture apparatus (Moor \& Mühlethaler, 1963) and examined in a Tesla BS 500 electron microscope.

\section{RESULTS \\ Optimal conditions for reversion}

The liquid medium YNBG osmotically stabilized with $0.6 \mathrm{M}-\mathrm{KCl}$ promoted polar growth of protoplasts and formation of fibrillar nets on their surfaces (see also Nečas \& Svoboda, 1981). In a medium containing 5 to $15 \%$ PEG instead of $0.6 \mathrm{M}-\mathrm{KCl}$, the protoplasts were rapidly disintegrated due to osmotic lysis. Higher PEG concentrations (20 to $40 \%$ ) protected the protoplasts osmotically and caused their aggregation into masses of varying sizes dependent on the initial pellet densities (Svoboda, 1978). The large aggregates could be disrupted by vigorous shaking and reaggregation could be prevented by plating the suspension on to Petri dishes, where, upon contact with the bottom, the small aggregates remained isolated.

The protoplasts did not show any evidence of development for 1 to $2 \mathrm{~h}$. Then they began to grow in a polar or isodiametric way, gradually becoming detached from the peripheries of the clusters. After a further 11-14 h the cytoplasm of some protoplasts thickened and, as revealed by light microscopy, the first distinct cell walls began to appear. The first signs of reversion were seen after 20 to $24 \mathrm{~h}$ of incubation as evidenced by budding of the regenerated protoplasts. In a concentration range of 20 to $40 \%$ PEG in the medium, the highest reversion frequency was observed in $35 \%$ PEG-media. In $20 \%$ PEG media reversion occurred only occasionally, the predominant form being the growing protoplasts. PEG at $40 \%$ in the medium also supported reversion but to a lesser extent. On the basis of these results media containing $35 \%$ PEG were employed in subsequent experiments.

The extent of protoplast growth was assessed quantitatively by measuring the increase in dry weight and the rate of synthesis of sedimentable polysaccharides. Optimal conditions for protoplast regeneration were provided by YNBG medium containing $2 \%(\mathrm{w} / \mathrm{v})$ glucose, in addition to $35 \%$ PEG (Table 1). This medium also gave the highest frequency of reversion, i.e. 20 to $30 \%$ of the protoplasts budded. A more exact estimate could not be made because of varying sizes of the aggregates and the asynchronous onset of reversion.

The occurrence of reversion was found to be a function of PEG concentration. When YNBG medium osmotically stabilized with $0.6 \mathrm{M}-\mathrm{KCl}$ was supplemented with increasing concentrations of PEG, reversion was observed only in media with PEG concentrations higher than $20 \%$. Lower concentrations (10 to $15 \%$ ), though inducing aggregation, did not support reversion; the protoplasts only developed in the way they did in liquid media without PEG.

Incubation temperature was not critical for the outcome of reversion between 20 to $30^{\circ} \mathrm{C}$. At $37^{\circ} \mathrm{C}$, however, the frequency of reversion was reduced by about half.

\section{Morphogenesis in protoplasts during reversion}

Morphology of the reverting protoplasts was related to the previous growth type. Protoplasts growing spherically were the first to revert (at 16 to $20 \mathrm{~h}$ of incubation; Fig. $1 a$ ); those showing polarised growth reverted after $20 \mathrm{~h}$ incubation (Fig. $1 \mathrm{~b}$ ). Restoration of normal morphogenesis 


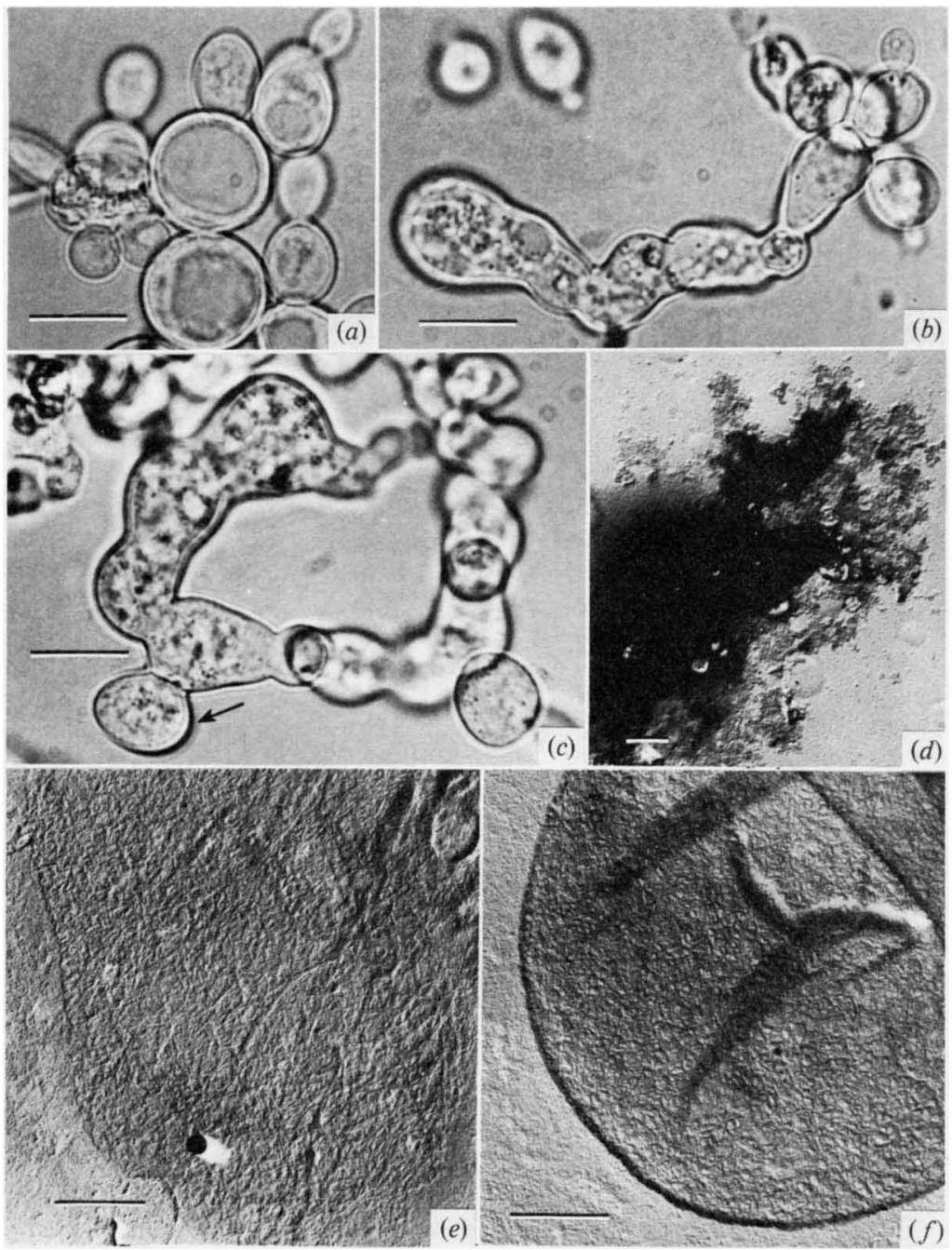

Fig. 1. Reversion and cell wall regeneration in $S$. cerevisiae protoplasts. (a) Spherical forms of regenerated protoplasts reverting to cells. The picture was taken after the protoplasts were cultured in $35 \%$ PEG-YNBG medium for $20 \mathrm{~h}$ at $28^{\circ} \mathrm{C}$. (b) Tubular form of regenerated protoplast reverting to cells after $24 \mathrm{~h}$ incubation in PEG-medium. (c) Tubular form of the growing protoplast after $30 \mathrm{~h}$ incubation in PEG-medium. Note the yeast-like cell (arrow) separated from the tube by a broad septum. The bar markers in $(a),(b)$ and $(c)$ represent $10 \mu \mathrm{m} .(d)$ Lysis of a freshly isolated protoplast on the formvar-coated grid. Only remnants of cytoplasmic components can be seen (shadow-cast preparation). (e) Fibrillar network isolated after the lysis of a protoplast grown in PEG-medium for $2 \mathrm{~h}$. $(f)$ Regenerating wall after $6 \mathrm{~h}$ incubation.'The fibrillar net is partly embedded in amorphous material. The bar markers in $(d),(e)$ and $(f)$ represent $1 \mu \mathrm{m}$.

occurred via one or more intermediate spherical or ellipsoidal forms. Non-reverting protoplasts grew into long tubular bodies that persisted for a few days before lysis. Occasionally, these protoplasts produced yeast-like forms which were unable to proliferate (Fig. 1c). Reverting protoplasts were discernible only at the periphery of the aggregates, while the centre consisted of a tangle of growing forms.

The process of reversion was not dependent on the presence of PEG in the medium. Protoplasts pre-cultured in PEG-medium for 8 to $12 \mathrm{~h}$ would then revert to cells even in the absence of PEG. 


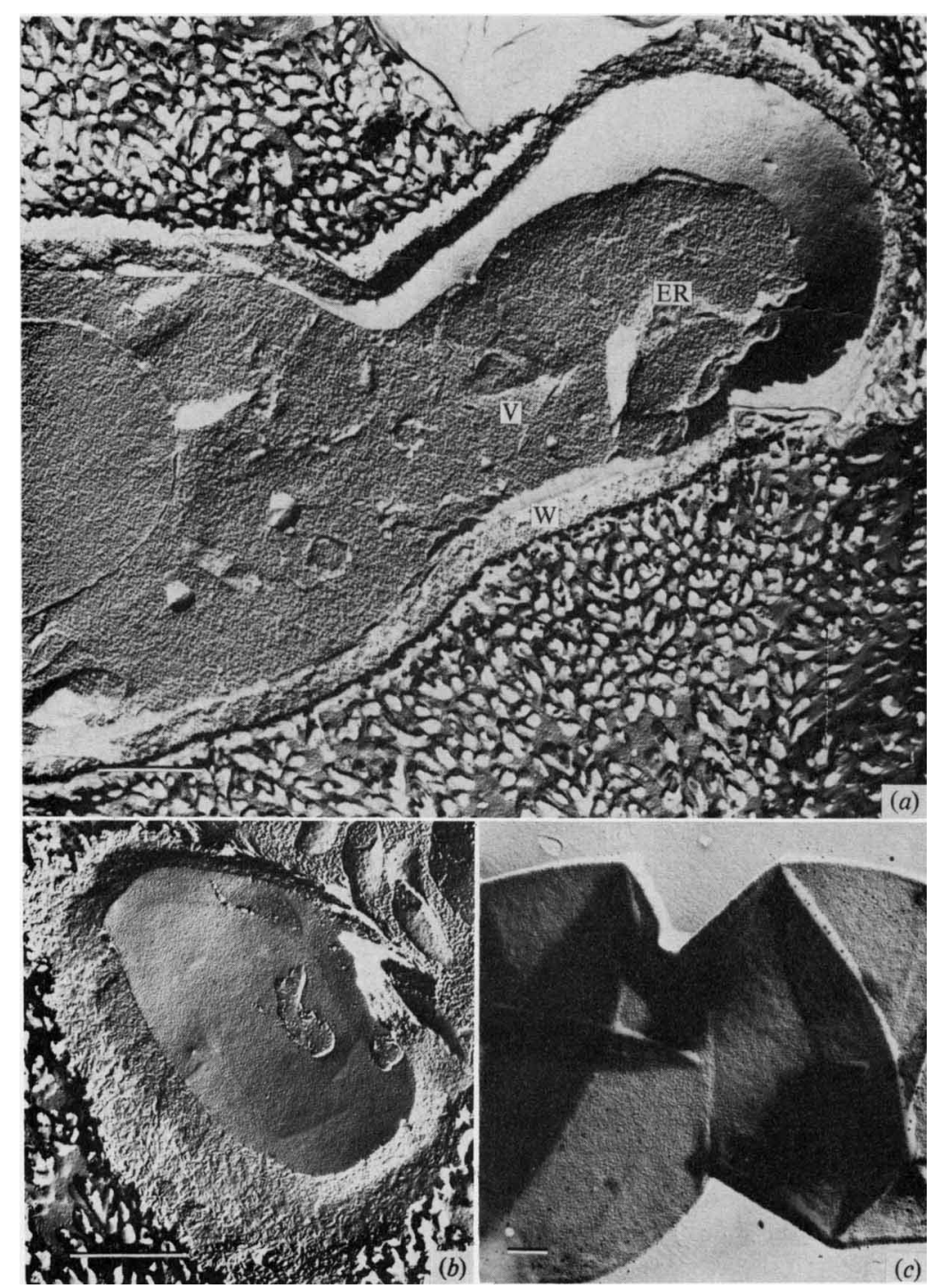

Fig. 2. Cell wall regeneration in $S$. cerevisiae protoplasts. (a) Freeze-etch micrograph of a protoplast grown in PEG-medium for $8 \mathrm{~h}$. A thick cell wall of granular appearance $(\mathrm{W})$, single vesicles (V) and the endoplasmic reticulum (ER) are indicated. (b) Regenerating protoplast at the same stage of development as in $(a)$, showing felt-like structure of the wall surface. $(c)$ Shadow-cast preparation of a regenerated wall isolated from a protoplast grown in PEG-medium for $18 \mathrm{~h}$. The fibrillar texture is still apparent. The bar markers represent $1 \mu \mathrm{m}$.

\section{Cell wall formation}

Freshly prepared protoplasts were free of any observable remnants of the cell wall (Fig. $1 d$ ). The first stage of the cell wall regeneration process was characterized by thin fibrillar networks (Fig. $1 e$ ) which appeared after 1 to $2 \mathrm{~h}$ of incubation in PEG-medium. The fine texture of the net indicated the presence of some cementing material in interfibrillar spaces. As incubation continued, the cell wall layer gradually increased in thickness but the fibrillar relief was preserved (Fig. 1f). On freeze-etched replicas the fractured cell wall showed a very coarse structure (Fig. 2a). Occasionally an exposed outer surface had a felt-like appearance (Fig. $2 b$ ). The regenerated wall in the period before reversion differed from the normal wall in that it had a fibrillar surface (Fig. 2c) and was thicker (Fig. 3a). 


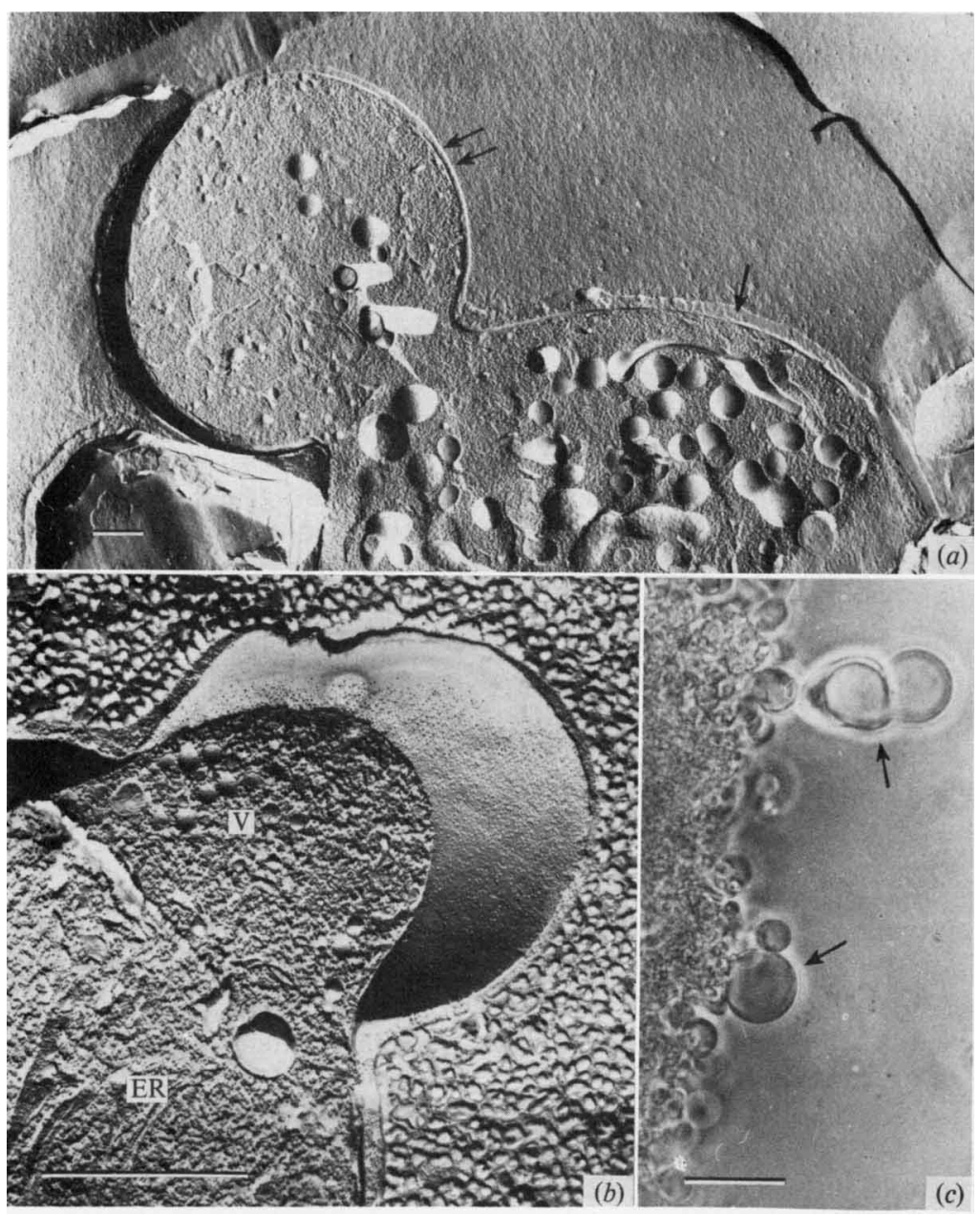

Fig. 3. Reverting protoplasts of $S$. cerevisiae. (a) Freeze-etch micrograph of a reverting protoplast. The bud contains several small vesicles and endoplasmic reticulum membranes. Note the difference in thickness between the regenerated wall (arrow) and the bud wall (double arrow). (b) Budding of a regenerated protoplast. Note the vesicles (V) and endoplasmic reticulum cisternae (ER). No signs of exocytosis are seen. The bar markers in $(a)$ and $(b)$ represent $1 \mu \mathrm{m}$. (c) Growing protoplasts (arrows) at the periphery of aggregated mixture of auxotrophic (non-growing) protoplasts. The bar marker represents $10 \mu \mathrm{m}$.

\section{Table 1. Cell wall synthesis and increase of dry weight of protoplasts during their incubation in $P E G$ media}

Protoplasts of $S$. cerevisiae 21-4-59 were cultured at $28^{\circ} \mathrm{C}$ in YNBG and YEG media containing $35 \%$ PEG 4000. The values were determined as indicated in Methods. Each value (with S.D.) is the mean of six measurements from three different experiments, each made in duplicate.

Medium

YNBG (1\% glucose)

YNBG $(2 \%$ glucose $)$

YNBG (5\% glucose)

YEG (2\% glucose)

$$
10^{-6} \times \text { Increase in }
$$

sedimentable polysaccharides $(\mathrm{g})^{*}$

$$
\begin{array}{r}
103.3 \pm 6.2 \\
168.9 \pm 7 \cdot 3 \\
143.0 \pm 5.6 \\
55.6 \pm 6.3
\end{array}
$$

$10^{-4} \times$ Increase in dry wt (g) $\dagger$

$$
\begin{array}{r}
8.1 \pm 1 \cdot 1 \\
12.2 \pm 1 \cdot 1 \\
8.3 \pm 0.9 \\
4.1 \pm 0.8
\end{array}
$$

* An absolute increase of sedimentable polysaccharides (in $\mu \mathrm{g}$ of glucose) during $6 \mathrm{~h}$ incubation of $7 \times 10^{6}$ protoplasts.

$\dagger$ An absolute increase of dry weight during $22 \mathrm{~h}$ incubation of $7 \times 10^{6}$ protoplasts. 
The cytoplasm of growing protoplasts, like that of intact cells, included only a few organelles. Endoplasmic reticulum cisternae were frequently observed under the plasma membrane (Fig. $2 a$ ) and groups of cisternae could also be seen in the cytoplasm (Fig. $3 b$ ). Vesicles thought to be involved in cell wall synthesis were rarely located close to the regenerating wall although they accumulated together with endoplasmic reticulum in growing buds (Fig. $3 a, b$ ).

The plasma membrane showed the usual structure characterized by densely packed intramembrane particles and short direct invaginations. (Fig. 2b). This structural arrangement was seen from the very beginning of cultivation and was different from that found in protoplasts cultured in liquid media (Nečas \& Svoboda, 1976).

\section{Reversion of fused protoplasts}

Protoplasts from auxotrophic strains 55R5-3C and 9 did not develop when suspended separately in PEG-minimal medium, or when a mixture of the two was incubated in minimal medium without PEG, indicating good stability of the genetic markers. When this complementary mixture was incubated in PEG-minimal medium, single growing bodies were observed at the periphery of the aggregates (Fig. $3 c$ ). Later, the bodies reverted to normal cells. On transfer to the minimal medium agar plates, the reverted cells retained their prototrophic character. Cytological and genetic analyses proved that the cells were hybrids with the same characteristics as those produced by the two-step procedure described previously (Svoboda, 1978). The same results were obtained when the protoplasts were first treated with PEG solution (Svoboda, 1978) and then transferred into the PEG-minimal medium. Fusion frequency, roughly estimated to be $10^{-3}$, was similar in both experimental procedures.

\section{DISCUSSION}

Media containing PEG provided good conditions for cell wall regeneration in protoplasts of $S$. cerevisiae and hence for their reversion to cells. Similar results were obtained with Schizosaccharomyces pombe, Candida albicans, Zygosaccharomyces rouxii and Trigonopsis variabilis (unpublished results). The results were generally similar to those obtained earlier in gelatin and in agar media (Nečas, 1961; Svoboda, 1966).

The question arises as to the role of PEG during cell wall regeneration. The function of solid media during cell wall regeneration has been explained by their ability to prevent soluble cell wall material from diffusing away from the protoplast surface (Nečas, 1971). The material retained is thus made available for enzyme-organized or auto-organized assembly of the complete cell wall (Nečas \& Svoboda, 1981). Liquid media, on the other hand, provide conditions only for formation of the fibrillar component (Eddy \& Williamson, 1959; Kopecká et $a l ., 1967$ ), because they allow soluble glycoproteins to be released into the medium (Lampen, 1968; Svoboda \& Nečas, 1970). The function of PEG during wall regeneration could be similar to that of gel media: the high viscosity of PEG and the number of hydrogen bonds formed between molecules make the diffusion of wall glycoproteins (mol. wt $10^{5}$; Ballou, 1976) difficult, thus increasing their accumulation at the protoplast surface. The relationship found in our experiments between reversion frequency and PEG concentration in media would support this idea.

Another explanation of the mechanism of PEG action on wall regeneration is based on the fact that PEG induces protoplast aggregation. Narrow spaces between the surfaces of aggregated protoplasts may easily be filled with the wall material and thus become the 'starting points' of complete cell wall assembly. The present observations showed that, although protoplast aggregation could be induced by adding 10 to $15 \%$ PEG to the medium, reversion to cells occurred only at concentrations of $20 \%$ and higher, which suggests that mere aggregation alone may not induce cell wall assembly.

Finally, the role of PEG should also be considered in terms of its effect on protoplast structure. The dehydrating effect of PEG 'preserves' the plasma membrane in such a way that its structure, especially the invaginations, is maintained in the same state as in normal cells or in protoplasts reverting in gelatin (Nečas \& Svoboda, 1976). This would suggest that a certain optimal arrange- 
ment of the plasma membrane produced in PEG or gels is required for the synthesis and assembly of a complete cell wall. Recently, a similar stabilizing effect of PEG.has been reported in another experimental system, cellulose synthesis in cotton fibres (Carpita \& Delmer, 1980).

The cryoprotective properties of PEG facilitated good preservation of the ultrastructure of the regenerating protoplasts, as seen on freeze-etched replicas. Vesicles and endoplasmic reticulum membranes in the cytoplasm of regenerating protoplasts and in the buds are evidently parts of the phenomenon of reversion (Nečas \& Svoboda, 1976). The exposed faces of the plasma membrane lacked exocytotic activity, indicating either that the process of exocytosis in protoplasts is very rapid or that even a short centrifugation of protoplasts before freezing may impair the intimate relationship between the plasma membrane and vesicles.

From our results it appears that the media supplemented with PEG have several advantages. They facilitate regeneration of the cell wall in protoplasts, a step necessary for the restoration of cell morphogenesis. They induce protoplast fusion and provide selective conditions for development of hybrid protoplasts. The dehydrating effect of PEG aids freeze-etch studies of growing and reverting protoplasts. However, the uncontrolled sizes of aggregates which affect the frequency of reversion and its accurate estimation present the main drawback to a broader application of PEG media.

We wish to thank Professor O. Nečas and Dr Z. Doležalová for their helpful comments and V. Janischová and J. Krobauer for technical assistance.

\section{REFERENCES}

Ballou, C. (1976). Structure and biosynthesis of the mannan component of the yeast cell envelope. Advances in Microbial Physiology 14, 93-158.

Carpita, N. C. \& Delmer, D. P. (1980). Protection of cellulose synthesis in detached cotton fibers by polyethylene glycol. Plant Physiology 66, 911-916.

Chung, C. W. \& Nickerson, W. J. (1954). Polysaccharide synthesis in growing yeasts. Journal of Biological Chemistry 208, 395-407.

DARLING, S. (1976). Polyethylene glycol-induced reversion of yeast protoplasts in liquid medium. Yeast Newsletter 25, 5-6.

EdDY, A. A. \& Williamson, D. H. (1959). Formation of aberrant cell walls and of spores by growing yeast protoplasts. Nature, London 183, 1101-1104.

KOPECKÁ, M. \& FARKAš, V. (1979). RNA synthesis and the formation of the cell wall. Effect of lomofungin on regenerating protoplasts of Saccharomyces cerevisiae. Journal of General Microbiology 110, 453-463.

Kopecká, M., ČtVrtníček, O. \& NeČas, O. (1967). Formation and properties of the fibrillar network formed in yeast protoplasts as the first step of biosynthesis of the cell wall. In Symposium über Hefeprotoplasten, pp. 73-76. Edited by R. Müller. Berlin: Akademie Verlag.

LAMPEN, J. O. (1968). External enzymes of yeast: their nature and formation. Antonie van Leeuwenhoek 34, $1-18$

LEUPOLD, U. (1955). Versuche zur genetischen Klassi- fizierung adeninabhängiger Mutanten von Schizosaccharomyces pombe. Archiv der Julius Klaus-Stiftung für Vererbungsforschung, Sozialanthropologie und Rassenhygiene 30, 506-516.

MoOR, H. \& MÜHLETHALER, K. (1963). Fine structure of frozen-etched yeast cells. Journal of Cell Biology 17, 609-628.

NEČAS, O. (1961). Physical conditions as important factors for the regeneration of naked yeast protoplasts. Nature, London 192, 1664-1665.

NEČAS, O. (1971). Cell wall synthesis in yeast protoplasts. Bacteriological Reviews 35, 149-170.

NEČAS, O. \& SvOBODA, A. (1976). Regeneration of yeast protoplasts. A freeze-etching study. Zeitschrift für allgemeine Mikrobiologie 16, 615-625.

NEČAS, O. \& SvobOdA, A. (1981). Morphogenesis in protoplasts. In Yeast Cell Envelopes - Biochemistry, Biophysics, and Ultrastructure, pp. 105-127. Edited by W. N. Arnold. Boca Raton: CRC Press.

SVOBODA, A. (1966). Regeneration of yeast protoplasts in agar gels. Experimental Cell Research 44, 640-642.

SvoBODA, A. (1978). Fusion of yeast protoplasts induced by polyethylene glycol. Journal of General Microbiology 109, 169-175.

SvoBodA, A. \& NEČAs, O. (1970). Experimental decoupling in the synthesis of fibrillar and amorphous components during regeneration of the cell wall of Saccharomyces cerevisiae protoplasts. In Yeast Protoplasts, pp. 211-215. Edited by O. Nečas \& A. Svoboda. Brno: J. E. Purkyně University. 